\title{
ACE inhibitors after myocardial infarction: selection and treatment for all
}

\author{
Andrew J S Coats
}

Angiotensin converting enzyme (ACE) inhibitors can reduce mortality in patients recovering from a myocardial infarction. In addition they improve prognosis in all stages of chronic heart failure and delay the progression of asymptomatic left ventricular dysfunction. They may also reduce the rate of reinfarction.

The evidence for these statements is based on large well conducted trials that give definitive answers about treatment effects on mortality. Controversy continues, however, about the best way to use this information in clinical practice. Recent correspondence in the Lancet $^{1-3}$ and two papers and the accompanying viewpoint in this issue of the British Heart fournal amply demonstrate the differences of opinion (see pages 466, 470, and 397). Why is the cardiological community arguing rather than congratulating itself on the timely and definitive data on important treatment options? The reasons are that individual trials test only a particular strategy in a particular population and, even before the ink is dry, arguments start about subgroups, extrapolation of results, and alternative strategies.

The post-myocardial infarction (MI) studies tested two distinct strategies: that of treating highly selected high risk patients for long periods ( $\mathrm{SAVE}^{4}$ and $\mathrm{AIRE}^{5}$ ) or that of treating "all-comers" for short periods but starting early (CONSENSUS $\mathrm{II},{ }^{6}$ GISSI- $3{ }^{7}$ and the recently published (18 March) ISIS-4 trial (Lancet 1995;345:669-85)). Much has been made of the fact that the first strategy gave a greater absolute risk reduction but this is as expected given the higher risk populations and longer duration of treatment. The success of the first strategy does not lessen that of studies that tested the more difficult question-that of treatment in the generality of patients in the short but crucial postinfarction period. The safety and further significant reduction in mortality shown in the short-term studies open up the possibility of applying both strategies of treatment to benefit the largest possible number of eligible patients to the fullest possible extent.
Are AGE inhibitors safe in the first day? In SAVE and AIRE patients did not start active treatment until several days after the index infarction. Though this allowed greater patient stability and allayed some fears about interfering with the "healing" process, it does mean that a substantial part of the post infarction mortality cannot be consideredthat is, those patients who die in the first 72 hours. This means that the results of these trials do not apply to all patients with myocardial infarction. Three trials have assessed mortality after early post infarction ACE inhibition. One study was equivocal (CONSENSUS II) and the other two (the two largest trials overall, GISSI-3 and ISIS-4) showed a significant net reduction in mortality, seen both in those with transient heart failure in the coronary care unit, and to a lesser extent in those without. ${ }^{3}$ These studies showed that it was safe to give ACE inhibition within the first 24 hours in most patients. There was an excess of hypotension and renal impairment, as has been seen in most other trials of ACE inhibitors in heart failure. The net reduction in mortality, however, shows that these effects are small compared with the benefits. We do not know whether a later start would have avoided the side effects while preserving the benefits. We do know, however, that treatment would not have been available to the patients who died in the first few days. These patients account for many of those who die within the first year, and this group contains a high proportion of the high risk patients with heart failure shown to benefit particularly from ACE inhibition.

Hypotension is the side effect that has caused the most concern. Although in GISSI-3 more patients treated with ACE inhibitor had hypotension than the control group, the death rate was higher in those who were spontaneously hypotensive in the control group. Thus a fall in blood pressure induced by an ACE inhibitor is not as adverse a sign as a spontaneous fall. One hundred and two (29\%) of 351 hypotensive patients in the control group died and $145(17 \%)$ of 852 hypotensive patients in the lisinopril group. 
It is not valid to compare the total number of deaths (102 with 145) without taking into account the different numbers of patients at risk. The death rate in the equivalent group of control patients who would have become hypotensive had they been given lisinopril cannot be determined because they are not identifiable. It is likely, however, that they are patients with borderline hypotension who are already at substantially increased risk. The total number of deaths in people who became hypotensive after lisinopril can be roughly compared, however, with what we might have expected. If we assume that the groups were well randomised, the additive effect of lisinopril on hypotension in the whole trial was an extra 501 hypotensive subjects of whom 43 died $(8 \cdot 6 \%)$. These 501 patients group were likely to be at an increased risk even without the ACE inhibitor because they would have included all the patients with borderline hypotension. We can only estimate mortality in these 501 people. Taking three estimates of 6 week mortality-that of the spontaneously hypotensive $(29 \%)$, that of the total trial population $(6.7 \%)$, or midway between the two $(18 \%)$-we can calculate the deaths in the relevant control group compared with the groups with lisinopril induced hypotension as 145 deaths, 34 deaths, or 90 deaths respectively. This compares with the observed result of 43 deaths, so the effect of lisinopril lies somewhere between a saving of 102 lives and a loss of nine. Given that the overall benefit of lisinopril is 76 lives saved, even the most pessimistic estimate of nine deaths that were lisinopril induced and hypotension related is acceptable. The more likely estimate is 90 deaths which indicates that about 47 lives were saved even among those with lisinopril induced hypotension.

The recently published CATS trials in patients with anterior MI also showed the safety of early ACE inhibition and indeed confirmed a reduction in the progression to heart failure in this group. ${ }^{8}$ In addition the ISIS-4 pilot study showed that the haemodynamic profile of early ACE inhibitor-induced hypotension was predominantly a vasodilator response with an increase in cardiac output and stroke volume that was maintained beyond the end of active treatment. ${ }^{9}$ Early hypotension in inself does not preclude a good outcome.

Despite the criticisms that not many lives were saved in GISSI-3 and ISIS-4, 5-8 lives saved per thousand by as little as $4-6$ weeks of treatment is a substantial and worthwhile gain, especially as it is a widely applicable, inexpensive treatment that does not require extensive investigation and can be given immediately. In addition patients who would never have satisfied the criteria of either SAVE or AIRE, either because they died too early or did not fulfil their entry criteria, can benefit from early ACE inhibition.

All trials are different, and each gives a different window on the truth. Where there is conflict between positive trials, it is best to compromise, and base treatments on the best composite of the available information. This would, in my opinion, involve treating all infarct survivors as potential ISIS-4 or GISSI-3 patients who would benefit from early ACE inhibition, and then after the first few weeks those who are similar to the patients of AIRE (clinical heart failure at some stage) or SAVE (significant left ventricular dysfunction) could continue on treatment long term and the others could stop. In the same way, after the first few years those survivors who still show left ventricular dysfunction or heart failure become like the SOLVD (studies of left ventricular dysfunction $)^{10}$ and CONSENSUS ${ }^{11}$ cohorts and should remain on ACE inhibition.

1 Ball SG, Reynolds GW, Murray GD. ACE inhibitors after myocardial infarction. Lancet 1994;343:1632.

2 myocardial infarction. Lancet $1994,343.1632$. myocardial infarction. Lancet 1994;343:1632-3.

Tognoni G. ACE inhibitors after myocardial infarction. Tognoni G. ACE inhibitors
Lancet 1994;343:1633-4.

4 Pfeffer MA, Braunwald E, Moyé LA, et al. Effect of captopril on mortality and morbidity in patients with left ventricular dysfunction after myocardial infarction. Results of the Survival and Ventricular Enlargement Trial. N Engl f Med 1992;327:669-77.

5 The Acute Infarction Ramipril Efficacy (AIRE) Study Investigators. Effect of ramipril on mortality and morbidity of survivors of acute myocardial infarction with clinical evidence of heart failure. Lancet 1993 342:821-8.

6 Swedberg K, Held P, Kjekshus J, et al. Effects of the early administration of enalapril on mortality in patients with acute myocardial infarction, results of the Cooperative New Scandinavian Enalapril Survival Study (CONSENSUS II). N Engl ₹ Med 1992;327:678-84.

7 Gruppo Italiano per lo Studio della Sopravvivenza nell'Infarto Micardio GISSI-3 effects of lisinopril and transdermal glyceryl trinitrate singly and together on 6 -week mortality and ventricular function after acute 6-week mortality and ventricular function infarction. Lancet 1994;343:1115-22.

8 Kingma JH, van Gilst WH, Peels $\mathrm{CH}$, et al. Acute intervention with captopril during thrombolysis in patients with first anterior myocardial infarction. Results from the Captopril and Thrombolysis Study (CATS). Eur Heart f 1994;15:898-907.

9 Pipilis A, Flather M, Collins R, et al. Hemodynamic effects of captopril and isosorbide mononitrate started early in acute myocardial infarction: a randomised placebo-controlled study. $\mathcal{F}$ Am Coll Cardiol 1993;21: 73-9.

10 Yusuf S, Pitt B, Davis CE. Treatment of chronic congestive heart failure. Reply. $N$ Engl $f$ Med 1992;326: 1220-1.

11 The CONSENSUS Trial Study Group. Effects of enalapril on mortality in severe congestive heart failure: results of the Cooperative North Scandinavian Enalapril Survival Study. N Engl F Med 1987;316:1429-35. 